Moroccan J. of Pure and Appl. Anal. (MJPAA)

Volume 7(1), 2021, Pages 173-181

ISSN: Online 2351-8227 - Print 2605-6364

DOI: $10.2478 / \mathrm{mjpaa}-2021-0013$

\title{
A look at nonexpansive mappings in non-Archimedean vector spaces
}

\author{
SAMIH LAZAIZ ${ }^{1}$
}

Aвstract. In a spherically complete ultrametric space every nonexpansive self-mapping $T$ has a fixed point $\bar{x}$ or a minimal invariant ball $B(\bar{x}, d(\bar{x}, T(\bar{x}))$. We show how we can approximate this fixed center $\bar{x}$ in a nonArchimedean vector space. And, we give a synthetic study for increasing mapping in non-Archimedean local fields.

Mathematics Subject Classification (2020). 47H09, 32P05

Key words and phrases. Fixed centers, Increasing mappings, Nonexpansive mappings, Pseudo-order, Ultrametric spaces.

\section{Introduction}

In the last 40 years, the theory of non-Archimedean analysis has taken a large place in mathematical research, this is motivated by several aspects, among them, there is a problem in quantum theory of the rupture of the Archimedean axiom (i.e. the use of real numbers) at the Planck scale. So, to have a better understanding at the Planck scale, it is quite natural to use p-adic numbers (see [6]). In addition, there are several applications of non-Archimedean analysis whether in mathematical physics, gravity, cosmology, biological systems, cognitive science and much more, for details one can consult the survey [4].

Received : May 13, 2020 - Accepted: November 25, 2020.

(C) The Author(s) 2020. This article is published with open access by Sidi Mohamed Ben Abdallah University.

${ }^{1}$ LaSMA Laboratory, Department of Mathematics, Faculty of Sciences Dhar El Mahraz, University Sidi Mohamed Ben Abdellah, Fes, Morocco.

e-mail: samih.lazaiz@usmba.ac.ma . 
Recall that a metric space $(M, d)$ is said to be ultrametric space (or, non-Archimedean space) if and only if

$$
d(x, z) \leq \max \{d(x, y) ; d(y, z)\},
$$

for any $x, y, z \in M$. Let $x \in M$ and $r>0$, we denote by $B(x ; r)$ the closed ball and by $B\left(x ; r^{-}\right)$ the open ball both of center $x$ and radius $r$.

A very important notion in non-Archimedean analysis which allows to carry many results of the classical functional analysis in the ultrametric case is the notion of spherical completeness.

Definition 1.1 ([19, Definition 1.1.4]). An ultrametric space $(M, d)$ is said to be spherically complete if every chain of balls in $M$ has nonempty intersection.

A mapping $T: M \rightarrow M$ is said to be nonexpansive if

$$
d(T(x), T(y)) \leq d(x, y) \text { for each } x, y \in M \text {. }
$$

Fixed point theory for nonexpansive mappings in ultrametric spaces find its root in the work of Petalas and Vidalis [10, Theorem 2], this theorem has been proved using Zorn's lemma. In 2012, Kirk and Shahzad [7, Theorem 4] revised the theorem by giving it a more illuminating form. Before we state this result recall that a ball $\hat{B}=B(x ; r)$ in $M$ is minimal $T$-invariant if $T(\hat{B}) \subseteq \hat{B}$ and $d(y, T(y))=r$ for all $y \in \hat{B}$.

Theorem 1.1 ([7, 10]). Suppose $M$ is a spherically complete ultrametric space and $T: M \rightarrow M$ is a nonexpansive mapping Then every ball of the form $B(x, d(x, T(x)))$ contains either a fixed point of $T$ or a minimal T-invariant ball.

The point $\bar{x} \in E$ such that $T(\bar{x})=\bar{x}$ or $B(\bar{x}, d(\bar{x}, T(\bar{x})))$ is a minimal $T$-invariant ball will be called fixed center. Moreover, this theorem has been generalized and extended by many authors see for instance $[1,11]$ and the references therein.

Remark 1.1. If $T$ is a nonexpansive mapping, then every ball of the form $B(x, d(x, T(x)))$ is $T$ invariant. Indeed, let $y \in B(x, d(x, T(x)))$ then $d(x, y) \leq d(x, T(x))$ we have by nonexpansivness

$$
d(T(y), T(x)) \leq d(x, T(x)) .
$$

Thus, $T(y) \in B(x, d(x, T(x)))$.

Many fixed point theorems for nonexpansive mappings are not constructive. There is no indication of how the fixed point by means of an iterative procedure can be approximated. From a practical point of view, it is important to be able to construct that fixed point. Berinde in [2], studied many of the approximate methods for nonexpansive mappings, namely Krasnoselskij iteration, the Mann iteration, the Ishikawa iteration and much more. The book is a good survey of the most used fixed point iteration procedures in classical Banach spaces. In a spherically complete ultrametric space, Prieß-Cramp and Ribenboim have used a transfinite Picard scheme to approximate the unique fixed point for strictly contractive mappings (i.e. mappings satisfying $d(T(x), T(y))<d(x, y)$ for each $x \neq y)$, for more details see [12, 13].

On the other hand, it is known that in the field of $p$-adic numbers $\mathbb{Q}_{p}$ there is no order which is compatible with both the group operation and the topology of $\mathbb{Q}_{p}$, see $[14$, Theorem 
3.4]. This property, which is frequently used in real analysis, presents difficulties for the construction of a theory of non-Archimedean ordered fields. In 1969 Monna [8] introduced the concept of pseudo-order in non-Archimedean complete field and showed that this binary relational system bahaves like an order (see also [9]). Later, Schikhof in [16, 17, 18] studied deeply this notion and introduced a several class of monotone mappings.

In section 2 of this paper, we introduce a transfinite Krasnoselskij method in order to approximate the fixed center for nonexpansive mappings. One can even consider this approach as another proof of Petalas-Vidalis' theorem [10, Theorem 2]. In section 3, we give a synthetic study of increasing mappings (i.e. mappings preserving pseudo-order) and we show that this class of mappings is fixed point free.

\section{Approximation theorem for nonexpansive mappings}

Let $\omega$ be the first transfinite ordinal and $\Omega$ be the first uncountable transfinite ordinal. Note that for each $\xi<\Omega, \xi$ is countable. For the use of transfinite induction in fixed point theory, one can consult [3] and references therein.

The following result is needed throughout this work, for the proof see [5, Proposition A.6, pp. 284].

Proposition 2.1. Suppose that a net $\left\{a_{\alpha}\right\}_{\alpha \in \Omega} \subseteq \mathbb{R}$ is bounded and either nonincreasing or nondecreasing. Then there exists $\alpha_{0} \in \Omega$ such that $a_{\alpha}=a_{\alpha_{0}}$ for all $\alpha \geq \alpha_{0}$.

Let $\mathbb{K}$ be a non-Archimedean local field (i.e. a field which is complete with respect to a nontrivial discrete non-Archimedean absolute value |.| and such that the residue class is finite). Let $E$ be a non-Archimedean vector space over $\mathbb{K}$, that is a $\mathbb{K}$-vector space endowed with a non-Archimedean norm $\|$.$\| :$

(1) $\|x\|=0$ if and only if $x=0$;

(2) $\|a x\|=|a|\|x\|$ for any $x \in E$ and any $a \in \mathbb{K}$;

(3) $\|x+y\| \leq \max \{\|x\| ;\|y\|\}$ for any $x ; y \in E$.

Let $E$ be a non-Archimedean spherically complete normed space and $T: E \rightarrow E$ a nonexpansive mapping. Then, there exists $\bar{x} \in E$ such that $T(\bar{x})=\bar{x}$ or $B(\bar{x},\|\bar{x}-T(\bar{x})\|)$ is a minimal $T$-invariant ball. Our objective is to indicate how to approximate such a "fixed center" $\bar{x}$. Note that since this point $\bar{x}$ is not necessary unique, our approach only approximate one of these points. The set of fixed centers will be denoted by $\operatorname{Fic}(T)$.

Let $k \in \mathbb{K}$ such that $0<|k| \leq 1$. Let $\mathcal{P}$ be the set of all families $\left(x_{\alpha}\right)_{\alpha \in \Omega} \subset E$ which satisfy for every $\alpha \in \Omega$, the following two conditions:

(i) $x_{\alpha+1}=(1-k) T\left(x_{\alpha}\right)+k x_{\alpha}$;

(ii) if $\mu$ is a limit ordinal, then $\left\|x_{\alpha}-x_{\mu}\right\| \leq\left\|x_{\mu}-T\left(x_{\mu}\right)\right\|$ for all $\mu<\alpha$.

Remark 2.1. This construction will be used to approach some fixed center $\bar{x}$ of $T$. Note that the proof of Theorem 2.1 below will show that $\mathcal{P}$ is a nonempty set.

Now, following [13] we introduce the following definition. 
Definition 2.1. We say that $\left(x_{\alpha}\right)_{\alpha \in \Omega} \subset E$ is a Krasnoselskij approximation of $\bar{x}$ if $\left(x_{\alpha}\right)_{\alpha \in \Omega} \in \mathcal{P}$ and

$$
\bigcap_{\alpha \in \Omega} B\left(x_{\alpha},\left\|x_{\alpha}-T\left(x_{\alpha}\right)\right\|\right)=B(\bar{x},\|\bar{x}-T(\bar{x})\|) .
$$

In fact, this definition captures the main idea of [13] and guarantees the extension of [13, Theorem 4] in the case of a non-Archimedean vector space. Note that if $\bar{x}$ is a fixed point of $T$, then $B(\bar{x},\|\bar{x}-T(\bar{x})\|)=\{\bar{x}\}$.

Before we state the main theorem it is worth mentioning that we can reach this point $\bar{x}$ by a finite steps of iterations, in this case we say that $x=\left(x_{\alpha}\right)_{\alpha<\lambda}$ reaches $\bar{x}$ for some finite ordinal $\lambda$.

Theorem 2.1. Let $E$ be a spherically complete non-Archimedean normed space. Assume that for any $\bar{x} \in$ Fic $(T)$ cannot be reached by any $\left(x_{\alpha}\right)_{\alpha \in \Omega} \in \mathcal{P}$ such that $x_{0} \neq \bar{x}$. Then for any $y \in E \backslash\{\bar{x}\}$ there exists a Krasnoselskij approximation $\left(x_{\alpha}\right)_{\alpha \in \Omega}$ of some $\bar{x} \in$ Fic $(T)$ such that $x_{0}=y$.

Proof. The idea of the proof is to construct a transfinite net $\left(x_{\alpha}\right)_{\alpha \in \Omega}$ where $\Omega$ is the first uncountable ordinal satisfying for each $\alpha \in \Omega$ :

$$
\begin{aligned}
& A_{1}(\alpha): x_{\alpha+1}=(1-k) T\left(x_{\alpha}\right)+k x_{\alpha} ; \\
& A_{2}(\alpha):\left\|x_{\alpha}-x_{\mu}\right\| \leq\left\|x_{\mu}-T\left(x_{\mu}\right)\right\| \text { whenever } \mu<\alpha .
\end{aligned}
$$

Let $x_{0} \in E$. For each $n$ in $\mathbb{N}$, set

$$
x_{n+1}=(1-k) T\left(x_{n}\right)+k x_{n} \quad \text { and } \quad B_{n}=B\left(x_{n},\left\|x_{n}-T\left(x_{n}\right)\right\|\right) .
$$

Since $B_{n}$ is $T$-invariant (see Remark 1.1) and

$$
|1-k| \leq \max \{1,|k|\} \leq 1,
$$

we have

$$
\begin{aligned}
\left\|x_{n+1}-x_{n}\right\| & =|1-k|\left\|T\left(x_{n}\right)-x_{n}\right\| \\
& \leq\left\|T\left(x_{n}\right)-x_{n}\right\| .
\end{aligned}
$$

Then $x_{n+1}$ and $T\left(x_{n+1}\right)$ are in $B_{n}$. Thus

$$
\begin{aligned}
\left\|x_{n+1}-T\left(x_{n+1}\right)\right\| & \leq \max \left\{\left\|x_{n+1}-x_{n}\right\|,\left\|x_{n}-T\left(x_{n+1}\right)\right\|\right. \\
& \leq\left\|x_{n}-T\left(x_{n}\right)\right\| .
\end{aligned}
$$

In addition, $B_{n+1} \subseteq B_{n}$ for all $n \in \mathbb{N}$. Indeed, let $y \in B_{n+1}$ so we have

$$
\left\|x_{n+1}-y\right\| \leq\left\|x_{n+1}-T\left(x_{n+1}\right)\right\|
$$

thus,

$$
\begin{aligned}
\left\|x_{n}-y\right\| & \leq \max \left\{\left\|x_{n}-x_{n+1}\right\|,\left\|x_{n+1}-y\right\|\right\} \\
& \leq\left\|x_{n}-T\left(x_{n}\right)\right\| .
\end{aligned}
$$

That is $B_{n+1} \subseteq B_{n}$ for all $n$. Then we get $B_{m} \subseteq B_{n}$ for all $n \leq m$.

This implies that $\left\{B_{n}\right\}_{n}$ is a descending chain of balls. By spherical completness of $E$, we have $\bigcap_{n \in \mathbb{N}} B_{n} \neq \varnothing$. Let $z \in \bigcap_{n \in \mathbb{N}} B_{n}$, since $B_{n}$ is $T$-invariant we get

$$
\|z-T(z)\| \leq\left\|x_{n}-T\left(x_{n}\right)\right\|, \quad \text { for all } n \in \mathbb{N} \text {. }
$$


Now, if $z=T(z)$ or $B(z,\|z-T(z)\|)$ is minimal ball, we take $\bar{x}=z$. Otherwise choose

$$
x_{\omega}=z \in \bigcap_{n \in \mathbb{N}} B_{n} .
$$

Then $A_{1}(\omega)$ and $A_{2}(\omega)$ are satisfied.

Let $\beta \in \Omega$. Assume that the net $\left\{x_{\alpha}\right\}_{\alpha<\beta}$ has been defined. We need to define $x_{\beta}$ and show that the properties $A_{1}(\beta)$ and $A_{2}(\beta)$ hold.

If $\beta$ is not a limit ordinal, say $\beta=\alpha+1$, take $x_{\beta}=(1-k) T\left(x_{\alpha}\right)+k x_{\alpha}$. Then $A_{1}(\beta)$ and $A_{2}(\beta)$ hold.

Assume, $\beta$ is a limit ordinal. Then for each $\mu \leq \alpha<\beta$, we have by $A_{2}(\alpha)$

$$
\left\|x_{\alpha}-x_{\mu}\right\| \leq\left\|x_{\mu}-T\left(x_{\mu}\right)\right\|
$$

that is $B_{\alpha} \subseteq B_{\mu}$. Again, using the spherical completeness of $E$, we get

$$
\bigcap_{\alpha<\beta} B\left(x_{\alpha},\left\|x_{\alpha}-T\left(x_{\alpha}\right)\right\|\right) \neq \varnothing .
$$

As above, let $z \in \bigcap_{\alpha<\beta} B\left(x_{\alpha},\left\|x_{\alpha}-T\left(x_{\alpha}\right)\right\|\right)$. If $z=T(z)$ or $B(z,\|z-T(z)\|)$ is a minimal ball take $\bar{x}=z$. Otherwise choose

$$
x_{\beta} \in \bigcap_{\alpha<\beta} B\left(x_{\alpha},\left\|x_{\alpha}-T\left(x_{\alpha}\right)\right\|\right)
$$

then $A_{1}(\beta)$ and $A_{2}(\beta)$ are satisfied.

Thus, the net $\left(x_{\alpha}\right)_{\alpha \in \Omega}$ is well constructed. In addition we have for all $\mu<\alpha<\Omega$ :

$$
\left\|x_{\alpha}-x_{\mu}\right\| \leq\left\|x_{\mu}-T\left(x_{\mu}\right)\right\| \quad \text { and } \quad\left\|x_{\alpha}-T\left(x_{\mu}\right)\right\| \leq\left\|x_{\mu}-T\left(x_{\mu}\right)\right\|
$$

by $A_{2}(\alpha)$ we have the first inequality and the second inequality is due to the fact that every point of a ball is a centre. Then,

$$
\begin{aligned}
\left\|x_{\alpha}-T\left(x_{\alpha}\right)\right\| & \leq \max \left\{\left\|x_{\alpha}-T\left(x_{\mu}\right)\right\|,\left\|T\left(x_{\mu}\right)-T\left(x_{\alpha}\right)\right\|\right\} \\
& \leq\left\|x_{\mu}-T\left(x_{\mu}\right)\right\|
\end{aligned}
$$

Hence $\left\{\left\|x_{\alpha}-T\left(x_{\alpha}\right)\right\|\right\}_{\alpha \in \Omega}$ is a nonincreasing net. Using Proposition 2.1 there exist $\alpha_{0} \in \Omega$ such that $\left\|x_{\alpha}-T\left(x_{\alpha}\right)\right\|$ is constant for all $\alpha \geq \alpha_{0}$. From $A_{2}\left(\alpha_{0}+1\right)$, we have $B_{\alpha_{0}+1} \subseteq B_{\alpha_{0}}$ and since $\left\|x_{\alpha_{0}+1}-T\left(x_{\alpha_{0}+1}\right)\right\|=\left\|x_{\alpha_{0}}-T\left(x_{\alpha_{0}}\right)\right\|$ we get $B_{\alpha_{0}+1} \supseteq B_{\alpha_{0}}$, that is $B_{\alpha_{0}+1}=B_{\alpha_{0}}$. Let $r=\inf _{\alpha \leq \alpha_{0}}\left\|x_{\alpha}-T\left(x_{\alpha}\right)\right\|$ then $\bigcap_{\alpha \leq \alpha_{0}} B\left(x_{\alpha},\left\|x_{\alpha}-T\left(x_{\alpha}\right)\right\|\right)=B\left(x_{\alpha_{0}}, r\right)$. Again, by $A_{2}\left(\alpha_{0}\right)$ we have :

$$
\left\|x_{\alpha_{0}}-T\left(x_{\alpha_{0}}\right) \leq\right\| x_{\alpha}-T\left(x_{\alpha}\right) \|
$$

for all $\alpha \leq \alpha_{0}$, that is $\bar{x}=x_{\alpha_{0}}$. 


\section{Monotone mappings in non-Archimedean local fields}

For a prime number $p$ we denote by $\mathbb{Q}_{p}$ the non-Archimedean valued field of the $p$-adic numbers, by $\mathbb{Z}_{p}$ its valuation ring $\left\{x \in \mathbb{Q}_{p}:|x| \leq 1\right\}$, this is the typical example of a nonArchimedean local field.

Regarding a partially ordered relation on $\mathbb{Q}_{p}$ we know that we cannot introduce an order structure having natural properties. In fact, from [18, p. 67], see also [14, 15] it is not possible to define a partial ordering $\geq$ on $\mathbb{Q}_{p}$ satisfying :

(i) $1 \geq 0 \geq-1$

(ii) if $a \geq 0, b \geq 0$ then $a+b \geq 0$

(iii) if $a_{n} \geq 0$ for all $n, \lim _{n \rightarrow \infty} a_{n}=a$ then $a \geq 0$,

that is to say $\mathbb{Q}_{p}$ does not admits an order which is compatible with both the group operation + and the usual topology of $\mathbb{Q}_{p}$. To get an idea, we have the following surprising result.

Proposition 3.1 ([14, lemma 3.3]). In $\mathbb{Q}_{p}$, the serie $\sum n . n$ ! converges to -1 .

To avoid this, A. F. Monna [8, 9] introduced the concept of sides of zero in a complete nonArchimedean field, which allowed him to define "positive element", "sign of element" and "increasing function".

We recall here the important properties that we will use in the sequel. For a full expository, see [17, section 4].

Let $\mathbb{K}$ be a non-Archimedean local field, $\mathbb{K}^{\times}=\mathbb{K} \backslash\{0\}$ and $\mathbb{K}^{+}=\{x \in \mathbb{K}:|1-x|<1\}$. In [17], $\mathbb{K}^{+}$is called the group of positive elements of $\mathbb{K}$. The group of signs of elements of $\mathbb{K}$ is defined to be the group $\Sigma$ whose elements are multiplicative cosets of $\mathbb{K}^{+}$, i.e.

$$
\Sigma:=\left\{a \mathbb{K}^{+}: a \in \mathbb{K}^{\times}\right\} .
$$

\section{Remark 3.1.}

(i) $\Sigma$ is an infinite group. Moreover, if we apply the above process to the archimedean local field $\mathbb{R}$, we find the two sides of zero, namely $(0, \infty)$ and $(-\infty, 0)$.

(ii) Let $\sigma \in \Sigma$ and let $x, y \in \sigma$. Then, there exist $a \in \mathbb{K}^{\times}$and $u, v \in \mathbb{K}^{+}$such that

$$
\sigma=a \mathbb{K}^{+}, x=a u \text { and } y=a v \text {. }
$$

Then,

$$
\begin{aligned}
|x-y| & =|a u-a v| \\
& =|a||u-v| .
\end{aligned}
$$

Moreover, since $u, v \in \mathbb{K}^{+}$, we have

$$
\begin{aligned}
|u-v| & =|(u-1)+(1-v)| \\
& \leq \max \{|u-1|,|v-1|\} \\
& <1 .
\end{aligned}
$$

Thus, $|x-y|<|a|$. In addition, since $u \in \mathbb{K}^{+}$and $u=x a^{-1}$ we obtain

$$
\left|x a^{-1}-1\right|<1 \Leftrightarrow|x-a|<|a| .
$$


Then, $|a|=|x|$. Similarly $|a|=|y|$, so in particular we have $|x|=|y|$.

The following definition can be found in $[8,9,17]$.

Definition 3.1 (Pseudo-order). Let $x, y \in \mathbb{K}$ and $\sigma \in \Sigma$. We say that $x<_{\sigma} y$ (y is greater than $x$ in the sense of $\sigma$ ) if $y-x \in \sigma$.

The next property shows that this binary relation behaves like an order.

Proposition 3.2 ([18, Proposition 85.3]). Let $x, y, z \in \mathbb{K}$.

(i) If $x \neq y$ then $x<_{\sigma}$ y for precisely one $\sigma \in \Sigma$. If $x=y$ then $x<_{\sigma}$ y for no $\sigma \in \Sigma$.

(ii) If $x<_{\sigma} y$ for some $\sigma$ then $x+z<_{\sigma} y+z$.

(iii) If $x<_{\sigma} y, 0<_{\sigma^{\prime}} z$ for some $\sigma, \sigma^{\prime}$ in $\Sigma$, then $x z<_{\sigma \sigma^{\prime}} y z$.

With this in hand, Schikhof introduced the following definition.

Definition 3.2 ([18, Definition 24.6]). Let $X \subset \mathbb{K}, \sigma \in \Sigma$ and $T: X \rightarrow \mathbb{K}$. $T$ is said to be increasing if for each $x, y \in X$ with $x \neq y$ we have

$$
x<_{\sigma} y \Rightarrow T(x)<_{\sigma} T(y) .
$$

Note that if $T$ is an increasing mapping, then it is an isometry. Indeed, for each $x, y \in X$ with $x \neq y$ the logical implication (3.1) can be written as

$$
y-x \in \sigma \Rightarrow T(y)-T(x) \in \sigma,
$$

so using Remark $3.1(i i)$, we get $|T(x)-T(y)|=|x-y|$.

We have the following surprising result

Proposition 3.3. Let $X \subset \mathbb{K}$. Every increasing mapping $T: X \rightarrow X$ is an isomerty.

We use some idea of Prieß-Cramp example [11, Example 1] to prove the following remark.

Remark 3.2. The class of increasing mappings is a proper class of nonexpansive mappings. Indeed, let $X=\mathbb{Z}_{3}$ and define $T: X \rightarrow X$ by $T(x)=x^{2}+x-3$ for each $x \in X$, and let $\pi \in X$ such that

$$
|1-\pi|<|\pi|
$$

then

$$
\begin{aligned}
|T(x)-T(y)-\pi| & =\left|\left(y^{2}-x^{2}\right)+(y-x-\pi)\right| \\
& \leq \max \left\{\left|y^{2}-x^{2}\right|,|y-x-\pi|\right\} .
\end{aligned}
$$

One can choose $y=4$ and $x=3$ then $y-x \in \sigma=B\left(\pi,|\pi|^{-}\right)=\left\{z \in \mathbb{Q}_{3}:|z-\pi|<|\pi|\right\}$ (see [18] for more details), using the fact that all triangles are isosceles we get

$$
|T(y)-T(x)-\pi|=\left|y^{2}-x^{2}\right|=1
$$

that is $T(y)-T(x) \notin \sigma$. Furthemore it is shown that $T$ is a nonexpansive mapping.

Since non-Archimedean local fields are spherically complete, the following question arise naturally :

Question. Let $X \subset \mathbb{K}$. Does an increasing mapping from $X$ to itself has a fixed point? 
Unfortunately the answer to this question is negative. The following counter-example shows the deviation from "classical" analysis.

Example 3.1. Let $X=\mathbb{Q}_{p}$. Define $T: X \rightarrow X$ by $T(x)=x+1$ for each $x \in X$. Let $\sigma \in \Sigma$, then there exists $\pi \in \mathbb{Q}_{p}^{\times}$such that $\sigma=B\left(\pi,|\pi|^{-}\right)$.

We have to show that for each $\sigma \in \Sigma, T$ is increasing mapping, i.e.

$$
y-x \in B\left(\pi,|\pi|^{-}\right) \Rightarrow T(y)-T(x) \in B\left(\pi,|\pi|^{-}\right) .
$$

Indeedd, we have obviously

$$
\begin{aligned}
|T(x)-T(y)-\pi| & =|y-1-x+1-\pi| \\
& <|\pi| .
\end{aligned}
$$

Thus, $|T(x)-T(y)-\pi|<|\pi|$. Then $T$ is an increasing mapping from $X$ to itself. Now, using Proposition 3.3, $T$ is nonexpansive mappingand it is clear that $T$ is fixed point free mapping

\section{Acknowledgments}

I would like to thank the anonymous referees for their careful reading of our manuscript and their many thoughtful comments and suggestions.

\section{References}

[1] M. Babahmed, A. El Amrani, and S. Lazaiz. Fixed points and diametral sets for sequentially bounded mappings in orbital ultrametric spaces. Proyecciones (Antofagasta, On line), 39(2):481-493, 2020.

[2] V. Berinde. Iterative approximation of fixed points, volume 1912. Springer-Verlag Berlin Heidelberg, 2007.

[3] K. Chaira, A. Eladraoui, M. Kabil, and S. Lazaiz. Extension of kirk-saliga fixed point theorem in a metric space with a reflexive digraph. International Journal of Mathematics and Mathematical Sciences, 2018, 2018.

[4] B. Dragovich, A. Y. Khrennikov, S. V. Kozyrev, I. V. Volovich, and E. I. Zelenov. p-adic mathematical physics: the first 30 years. p-Adic Numbers, Ultrametric Analysis and Applications, 9(2):87-121, 2017.

[5] M. A. Khamsi and W. A. Kirk. An introduction to metric spaces and fixed point theory, volume 53. John Wiley \& Sons, 2011.

[6] A. Y. Khrennikov. non-Archimedean analysis: quantum paradoxes, dynamical systems and biological models, volume 427. Springer Science \& Business Media, 2013.

[7] W. A. Kirk and N. Shahzad. Some fixed point results in ultrametric spaces. Topology and its Applications, 159(15):3327-3334, 2012.

[8] A. F. Monna. Sur une classe de corps topologiques munis d'un pseudo-ordre. In Indagationes Mathematicae (Proceedings), volume 72, pages 364-375. North-Holland, 1969.

[9] A. F. Monna. Ordre, pseudo-ordre, primes et convexité. In Indagationes Mathematicae (Proceedings), volume 74, pages 181-190. Elsevier, 1971.

[10] C. Petalas and T. Vidalis. A fixed point theorem in non-Archimedean vector spaces. Proceedings of the American Mathematical Society, 118(3):819-821, 1993.

[11] S. Priess-Crampe. Fixed points and stable balls in ultrametric spaces. Results in Mathematics, 43(1-2):163-167, 2003.

[12] S. Priess-Crampe. Reaching fixed points as limits in subspaces. Journal of Geometry, 107(2):387-402, 2016.

[13] S. Priess-Crampe and P. Ribenboim. The approximation to a fixed point. J. Fixed Point Theory Appl., 14(1), 41-53 (2013).

[14] G. Rangan. On orderability of topological groups. International Journal of Mathematics and Mathematical Sciences, 8(4):747-754, 1985. 
[15] P. Ribenboim. The theory of classical valuations. Springer Science \& Business Media, 2012.

[16] W. H. Schikhof. non-Archimedean monotone functions. Groupe de travail d'analyse ultramtrique, http://www . numdam.org/item/GAU_1978-1979__6__A7_0/, Volume 6 (1978-1979), Talk no. 13, 8 p.

[17] W. H. Schikhof. p-adic monotone functions. https://hdl . handle .net/2066/57035, 1980.

[18] W. H. Schikhof. Ultrametric Calculus: an introduction to p-adic analysis, volume 4. Cambridge University Press, 2007.

[19] W. H. Schikhof. Locally convex spaces over non-Archimedean valued fields, volume 4. Cambridge University Press, 2010. 